EPJ Web of Conferences 111, 10003 (2016)

DOI: $10.1051 /$ epjconf/201611110003

(C) Owned by the authors, published by EDP Sciences, 2016

\title{
Sensitivity studies of spin cut-off models on fission fragment observables
}

\author{
L. Thulliez ${ }^{1,2, a}$, O. Litaize ${ }^{1}$, and O. Serot ${ }^{1}$ \\ ${ }^{1}$ CEA, DEN, DER, SPRC, F-13108 Saint Paul Lez Durance, France \\ ${ }^{2}$ CEA, DSM, Irfu, SPhN F-91191 Gif-sur-Yvette, France
}

\begin{abstract}
A fission fragment de-excitation code, FIFRELIN, is being developed at CEA Cadarache. It allows probing the characteristics of the prompt emitted particles, neutrons and gammas, during the de-excitation process of fully accelerated fission fragments. The knowledge of the initial states of the fragments is important to accurately reproduce the fission fragment observables. In this paper a sensitivity study of various spin cut-off models, completely defining the initial fission fragment angular momentum distribution has been performed. This study shows that the choice of the model has a significant impact on gamma observables such as spectrum and multiplicity and almost none on the neutron observables.
\end{abstract}

\section{Introduction}

During the fission fragment de-excitation, nuclei emit neutrons and gammas to loose their excitation energy and angular momentum in order to reach their ground state. The properties of those prompt emitted particles is of particular interest for nuclear reactors, for example to quantify the damage they can cause on the nuclear reactor vessel which has a direct impact on its lifetime. A Monte-Carlo computer code named FIFRELIN [1] [2] developed at CEA-Cadarache allows studying nuclear fission through the nuclear fission fragment de-excitation process. This is performed through a Monte-Carlo implementation of the so-called Hauser-Feshbach algorithm which takes into account the $\mathrm{n} / \gamma$ competition [2]. Practically the notion of nuclear realization is used as proposed for capture reactions in [3]. In this work the impact of nuclear models on fission observables, i.e. the outputs of the code, is investigated. By comparing the observables estimated by the code with the available experimental data, the relevance of the models can be inferred. If results related to major observables are in good agreement with available data, other calculated observables, which could not be measured, could be legitimately considered as pertinent. This confers to FIFRELIN a predictive power.

This paper will focus on the impact of the spin cut-off parameter models which defined the total angular momentum distribution from which the initial spins of the fragments are sampled. This entity mainly drives the characteristics of gammas such as their energy, their multiplicity and their spectrum. In order to perform this study, only the spin cut-off parameters of the simulation are tuned, the other parameters are unchanged. The spin cut-off models are tested in the case of the ${ }^{252} \mathrm{Cf}$ spontaneous fission.

\footnotetext{
a e-mail: loic.thulliez@cea.fr
} 


\section{Spin cut-off models involved in the initial spin distribution}

In order to perform the de-excitation of each fragment, their excitation energy $E^{*}$ and spin parity $J^{\pi}$ have to be assigned. The procedure to determine $\mathrm{E}^{*}$ is described in detail in [1]. An equal probability for parity is considered: $\mathrm{p}(\pi=+)=\mathrm{p}(\pi=-)=\frac{1}{2}$ The initial total angular momentum $J$, of the light (L) and heavy $(\mathrm{H})$ fragments, is sampled in the distribution given by equation (1).

$$
P_{L, H}(J)=\frac{(2 J+1)}{2 \sigma_{L, H}^{2}} \exp \left(-\frac{(J+1 / 2)^{2}}{2 \sigma_{L, H}^{2}}\right)
$$

where $\sigma_{L, H}^{2}$ is the spin cut-off parameter, related to the mean and the width of the distribution.

Four different models have been implemented in FIFRELIN for the spin cut-off determination. Each model has free parameters which are adjusted to reproduce the ${ }^{252} \mathrm{Cf}$ total prompt neutron multiplicity $\bar{v}=3.76$. The tested models are the following :

- Constant model, in which the light and the heavy fragment groups are described by a constant spin cut-off:

$$
\sigma_{L, H}=K_{L, H}
$$

$\mathrm{K}_{L}$ and $\mathrm{K}_{H}$ are two free parameters .

- Linear model, based on Armbruster experimental data [4], in which the spin cut-off of each mass group is linear as a function of the mass :

$$
\sigma_{L, H}=a_{L, H} A+b_{L, H}
$$

The coefficients $a_{L}$ and $a_{H}$ are the one from Armbruster while the $b_{L}$ and $b_{H}$ are simulation free parameters. This prescription is used to restrict the number of free parameters in the simulation.

- Inertia model, which was formulated by Bethe [5] depends on the mass and on the temperature of the nucleus. It takes into account shell effects through the Ignatyuk level density $a$ parameter [6] used to calculate the nucleus temperature T.

$$
\sigma_{\text {Inertia }}=\alpha_{\text {Inertia }} \sqrt{I_{\text {rig }} T}
$$

with $\alpha_{\text {Inertia }}$ a free parameter, $I_{\text {rig }}$ the moment of inertia of the nucleus assuming it as a pure rigid rotor and $\mathrm{T}$ the nucleus temperature. The temperature is calculated considering the nucleus as a Fermi gas and in taking the Ignatyuk prescription for the level density parameter $a$ calculation. Thus, the "Inertia" model lowers the number of free parameters by one contrary to the "Constant" and "Linear" models.

- Inertia+Shell model, which comes from observations following microscopic calculations [7] [8] and whom pointed out that the ratio $\frac{\sigma^{2}}{T}$ is not a constant but shows some shell effects, as already obtained from semi-empirical approaches [9] [10]. Thus the following spin cut-off can be formulated:

$$
\sigma_{\text {Inertia }+ \text { Shell }}=\alpha_{\text {Inertia }+ \text { Shell }} \sqrt{\frac{a}{\tilde{a}} I_{\text {rig }} T}
$$

with $\alpha_{\text {Inertia }+ \text { Shell }}$ a free parameter, $a$ the Ignatyuk level density parameter [6] and $\tilde{a}$ its asymptotic parameter. As in the previous model, the number of free parameters is one. 
The mean total angular momentum as a function of the pre-neutron fission fragment mass A obtained with the four models are plotted on Figure 1. In the region of high mass yields, i.e. around $A=108$ and $A=145$, the Constant model is a mean of the Inertia+Shell model. Consequently, one expects that those two models give approximately the same results. Figure 1 shows that Inertia and Inertia+Shell models do not have pronounced shell effects. This is due to the high initial energy given to primary fragments, which almost vanishes the shell effects in the level density parameter.

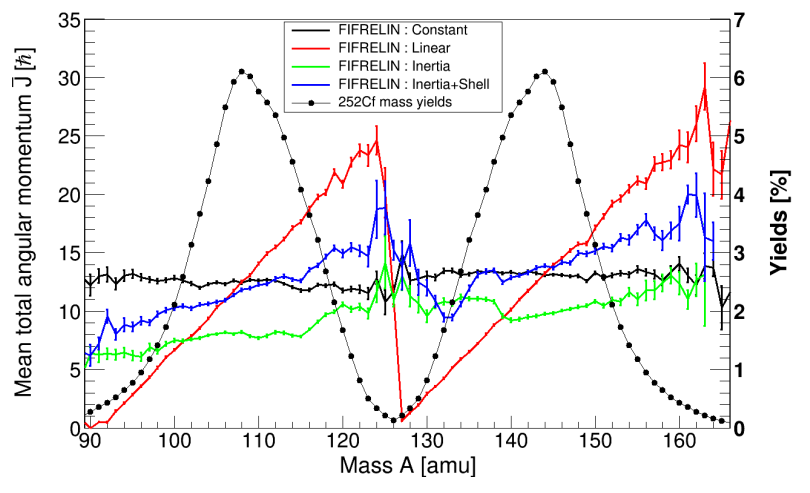

Figure 1. Mean Total angular momentum as a function of the pre-neutron fission fragment mass for the four tested models (left Y-axis) with the ${ }^{252} \mathrm{Cf}$ mass yields (right Y-axis).

\section{Impact of spin cut-off models on fission observables}

In this section, the four models are applied to the spontaneous fission of ${ }^{252} \mathrm{Cf}$, compared to neutron and gamma experimental data and discussed.

\subsection{Gamma observables}

Figure 2 shows the prompt fission gamma spectra (PFGS) obtained with different initial spin distributions and compared with experimental data [11] [12]. Below $1.2 \mathrm{MeV}$, the spectrum obtained with the Inertia model underestimates experimental data contrary to the three others, which slightly overestimate the data of about one gamma per fission per MeV. In the gamma energy range between 1.2 $\mathrm{MeV}$ and 8.0 MeV (right side of Figure 2) all models agree with experimental data.

The discrepancies below 1.2 MeV with the Inertia model are clearly explained from the averaged values of the sampled total angular momenta, $\bar{J}_{L}=8.00 \hbar$ and $\bar{J}_{H}=10.45 \hbar$, which are significantly lower than the ones obtained with the three other models (see Table 1). The relation between a high initial total angular momentum and a high emission of low energy gamma can be understood in term of $\mathrm{n} / \gamma$ competition. The higher the initial total angular momentum is, the higher in excitation energy the competition will occur in order to decrease the angular momentum of the fragment [2]. For example the competition starts around the neutron separation energy $S_{n}$ for low angular momenta $\left(J_{i} \approx 3 \hbar\right)$ and around $S_{n}+3 \mathrm{MeV}$ for high angular momenta $\left(J_{i} \approx 16 \hbar\right)$. The competition starts higher in energy in order to give the nucleus the right spins to allow a gamma transition from the continuum to the first discrete levels. Consequently, in the $\mathrm{n} / \gamma$ competition zone, the excess of angular momentum is expected to be mainly evacuated by a cascade of low energy gammas. 

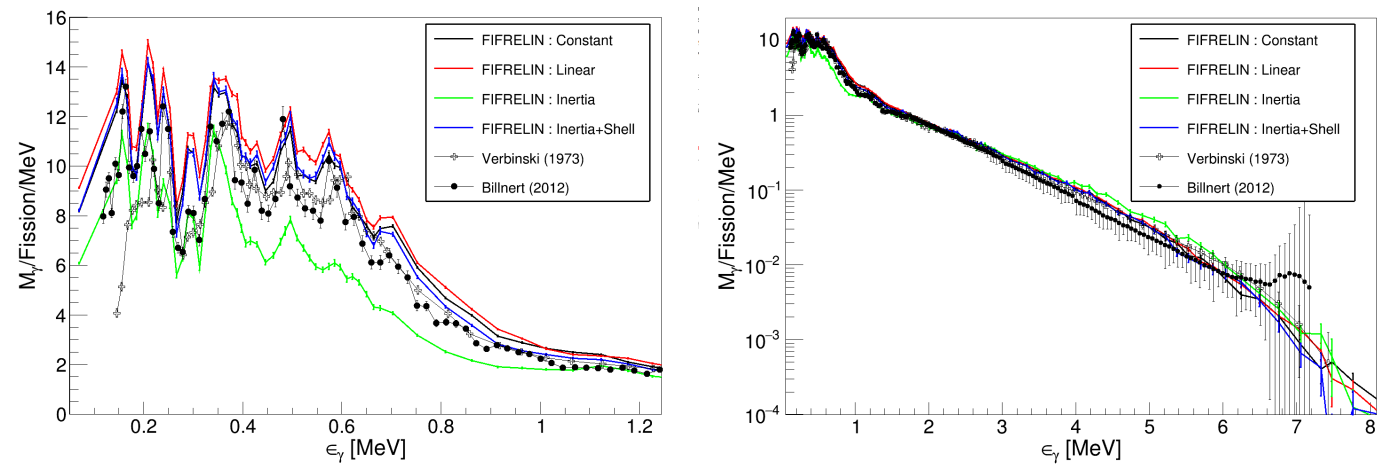

Figure 2. Prompt fission gamma spectra at low energy (left side) and up to $8 \mathrm{MeV}$ (right side) compared with experimental data [11] [12].

Table 1 presents the main relevant averaged gamma quantities obtained experimentally and with FIFRELIN calculations. When comparing experimental and simulated averaged gamma quantities, the experimental setup such as energy threshold or coincidence time have to be taken into account in FIFRELIN calculations. Practically, when the threshold increases, the mean gamma energy increases (due to a cut in the low energy part of the spectrum) and the average multiplicity decreases (preserving a constant total gamma energy). Otherwise the comparison is fortuitous as Albinsson shown on the gamma multiplicity as a function of fragment mass [13] and reported by Brunson [14]. In our case a $110 \mathrm{keV}$ threshold and $3 \mathrm{~ns}$ coincidence time are used, comparable with Billnert data. Table 1 shows that on average the Constant, Linear and Inertia+Shell spin cut-off models predict around one more gamma than experimental values and deposit an additional amount of $1 \mathrm{MeV}$ in the gamma channel.

Table 1. Comparison between experimental data citeVerbinski [12] and FIFRELIN calculations obtained with Billnert experimental parameters ( $\mathrm{E}_{\gamma}^{\text {threshold }}=110 \mathrm{keV} ; \mathrm{T}_{\text {coinc }}=3 \mathrm{~ns}$ ). In Verbinski experiment, parameters were $\mathrm{E}_{\gamma}^{\text {threshold }}=140 \mathrm{keV}$ and $\mathrm{T}_{\text {coinc }}=10 \mathrm{~ns}$, leading to a lower multiplicity $\bar{M}_{\gamma}$ and a higher average energy per quantum $\left\langle\epsilon_{\gamma}\right\rangle$.

\begin{tabular}{|c||c|c|c|c|c|}
\hline${ }^{252} \mathrm{Cf}$ & $\bar{J}_{L}$ & $\bar{J}_{H}$ & $\left\langle\epsilon_{\gamma}\right\rangle$ & $\bar{M}_{\gamma}$ & $\left\langle E_{\gamma}^{\text {tot }}\right\rangle$ \\
\hline Verbinski (1973) & $/$ & $/$ & $0.88 \pm 0.04$ & $7.80 \pm 0.3$ & $6.84 \pm 0.3$ \\
Billnert (2012) & $/$ & $/$ & $0.80 \pm 0.01$ & $8.30 \pm 0.08$ & $6.64 \pm 0.08$ \\
Constant & 12.39 & 13.18 & 0.81 & $9.40 \pm 0.02$ & $7.74 \pm 0.01$ \\
Linear & 13.17 & 12.54 & 0.80 & $9.99 \pm 0.02$ & $8.07 \pm 0.01$ \\
Inertia & 8.00 & 10.45 & 0.90 & $6.89 \pm 0.03$ & $6.29 \pm 0.02$ \\
Inertia+Shell & 12.22 & 13.96 & 0.80 & $9.46 \pm 0.03$ & $7.67 \pm 0.02$ \\
\hline
\end{tabular}

Figure 3 represents the average gamma multiplicity as a function of fission fragment mass. It is not well established that a saw-tooth shape can be expected. A detailed study of Albinsson for ${ }^{235} \mathrm{U}\left(\mathrm{n}_{t h}, \mathrm{f}\right)$ reaction [13] has shown that a flat or a saw-tooth behaviour can be obtained, depending on the experimental setup and gamma energy range. Even an inverse saw-tooth shape can be obtained. Here we simply show that a saw-tooth behaviour is directly obtained if the $\mathrm{J}_{R M S}$ of the initial spin distribution has a saw-tooth shape itself (Linear model). However an increase of the multiplicity is observed for heavy fragments as a function of mass whatever the used model. The expected saw-tooth 
behaviour of the Linear model is found but does not reproduce the Schmidt-Fabian data [15]. The three other models give results which have a less pronounced behaviour. However the Inertia model always underestimates the data, while the Constant and Inertia+Shell models are closer.

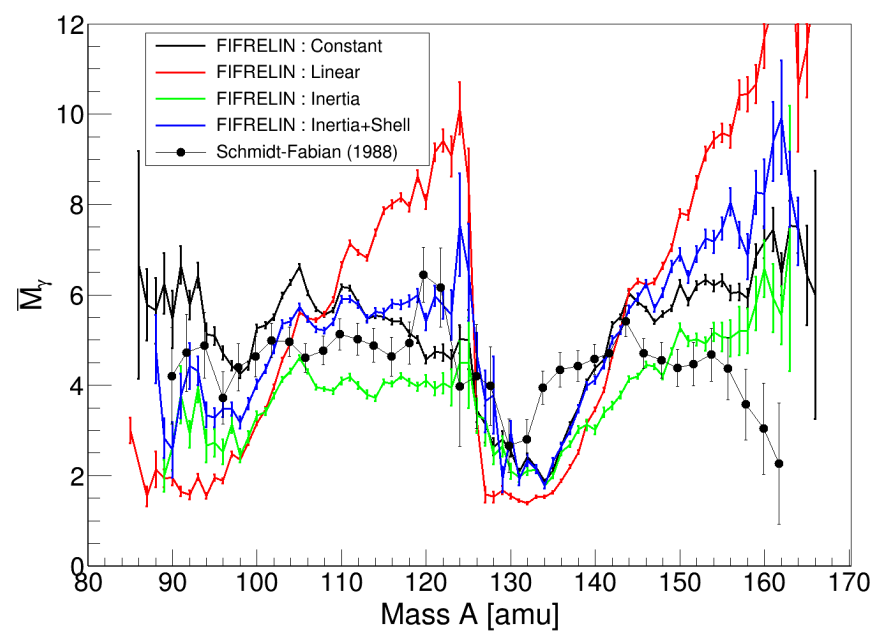

Figure 3. Comparison of the average gamma multiplicity as a function of the pre-neutron mass between SchmidtFabian data [15] and FIFRELIN calculations with different spin cut-off models.

In conclusion, the choice of the spin cut-off models for the initial total angular momenta has a great impact on gamma observables as expected. This study shows on one hand that the experimental parameters involved during the measurements must be taken into account in any calculations and on the other hand that for the moment no spin cut-off model allows reproducing the whole gamma observables. More experimental data are needed in order to confirm the previous one and to perform other verifications of the models on other fissioning nuclei.

\subsection{Neutron observables}

Figure 4 represents the neutron multiplicity as a function of mass and the prompt neutron spectra ratio to a Maxwellian $(\mathrm{T}=1.42 \mathrm{MeV})$, showing that there are no significant differences between the four tested models for those neutron observables, contrary to what could be expected due to a smaller $\mathrm{n} / \gamma$ competition zone in the case of the Inertia model.

In the left side of Figure 4, all models are in good agreement with the different experimental data sets. Nevertheless the Constant and Inertia+Shell models seem to better reproduce the data, especially for the very asymmetric fission. The neutron spectra represented on figure 4 are not different from one model to another. However at energy below $0.2 \mathrm{MeV}$ the Inertia and Inertia+Shell models seem to have some structures contrary to the two others. This could be a hint for further investigations. 

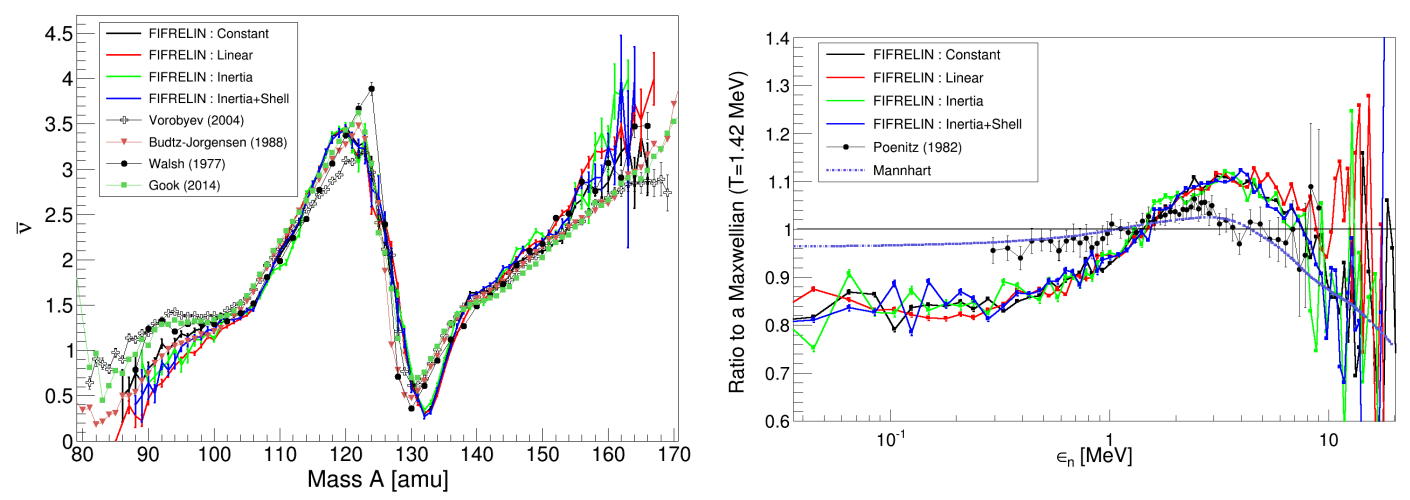

Figure 4. Comparison of neutron multiplicity as a function of mass (left side) and prompt fission neutron spectra ratio to a Maxwellian at $\mathrm{T}=1.42 \mathrm{MeV}$ (right side) between experimental data and FIFRELIN calculations performed with different spin cut-off models.

\section{Conclusion}

In this work the impact of spin cut-off models for initial spin distribution on fission observables was studied through the spontaneous fission of ${ }^{252} \mathrm{Cf}$. It has been shown that for the moment no model allows reproducing gamma observables accurately. However the Constant and the Inertia+Shell models seem to better reproduce experimental data than the others. The choice of the spin cut-off model has been shown to have no impact on neutron observables, and consequently does not softer the hard PFNS obtained with FIFRELIN. In order to try to improve the calculation of this observable, new energy sharing models are under investigation.

In perspective works, the presented spin cut-off models will be tested on other fissioning nuclei to validate these tendencies.

\section{References}

[1] O. Litaize and O. Serot, Phys. Rev. C 82, 054616 (2010)

[2] D. Regnier, Ph.D. thesis, Univeristy of Grenoble (2013)

[3] F. Becvar, Nucl Instrum. Methods Phys. Res., Sect. A 417(2-3), 434 (1998)

[4] P. Armbruster et al., Z. Naturforsch. 26 a, 512 (1971)

[5] H. A. Bethe, Phys. Rev. vol 50, 332 (1936)

[6] A. V. Ignatyuk et al., Sov. J. Nucl. Phys. Vol 21, 255 (1975)

[7] A. J. Koning, S. Hilaire and S. Goriely, Nucl. Phys. A 810, 13 (2008)

[8] S. Goriely, Nucl. Phys. A 605, 28 (1996)

[9] S. K. Kataria et al., Phys. Rev. C 18, 549 (1978)

[10] H. Vonach et al., Phys. Rev. C 38, 2541 (1988)

[11] V. V. Verbinski et al., Phys. Rev. C 7(3), 1173 (1973)

[12] R. Billnert et al., Phys. Rev. C 87(2), 246 (2013)

[13] H. Albinsson, Aktiebolagand Atomenergi Report AE-420 (1971)

[14] G. S. Brunson, Los alamos Report LA-9408-T (1982)

[15] C. Wagemans, The Nuclear Fission Process (CRC Press 1 edition, September 1991) 